PROF. ANDREW ZALESKY (Orcid ID : 0000-0003-2298-9908)

MS. TABINDA SARWAR (Orcid ID : 0000-0001-7313-5350)

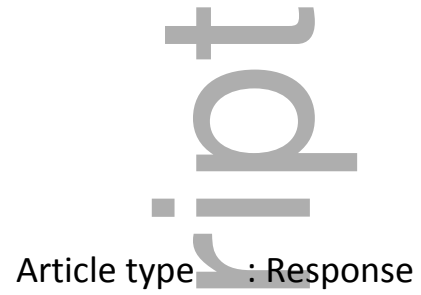

\title{
Response: A Cautionary Note on the Use of SIFT in Pathological Connectomes
}

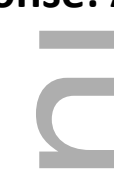

Andrew Zalesky ${ }^{1,2}$, Tabinda Sarwar $^{3}$, Ramamohanarao Kotagiri ${ }^{3}$

1 Department of Biomedical Engineering, The University of Melbourne, Victoria, 3010, Australia

2 Melbourne Neuropsychiatry Centre, The University of Melbourne, Victoria, 3010, Australia

3 Department of Computing and Information Systems, The University of Melbourne, Victoria 3010, Australia

Response to the Letter to the Editor by Smith and colleagues:

https://onlinelibrary.wiley.com/doi/full/10.1002/mrm.27916

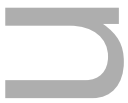

Corresponding author:

Associate Professor Andrew Zalesky

Melbourne Neuropsychiatry Centre

This is the author manuscript accepted for publication and has undergone full peer review but has not been through the copyediting, typesetting, pagination and proofreading process, which may lead to differences between this version and the Version of Record. Please cite this article as doi: 10.1002/MRM.28037

This article is protected by copyright. All rights reserved 
Level 3, Alan Gilbert Building

The University of Melbourne

Victoria 3010, AUSTRALIA

Email: azalesky@unimelb.edu.au

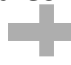

Number of Figures: 1

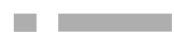

Number of Tables: $\quad 0$

Supplementary Figures: 0

Supplementary Tables: 0

We recently presented a hypothetical example (1) that demonstrates paradoxical behavior of the spherical-deconvolution informed filtering of tractograms (SIFT) method (2) in pathology. The Letter to the Editor by Smith and colleagues (3) contends that the paradoxical behavior of the SIFT method exemplified by our example can be remedied by: i) connection density normalization; and, ii) use of a more realistic number of streamlines. Here, we revisit our example and show that these two modifications do not alleviate the paradoxical conclusions that result when SIFT is used. Specifically, we demonstrate that the SIFT method augmented with the two modifications proposed by Smith and colleagues can cause paradoxical changes in connectivity, which lead to wrong conclusions about the location of pathological fibers. SIFT can cause normal fiber bundles without any pathology to show the same reduction in connectivity as pathological fibers. While we did not evaluate SIFT in our original investigation (1), and thus our conclusions and recommendations therein are unaffected by this critique, we welcome the opportunity to analyze our model in more detail here.

Model: Our fiber bundle phantom comprises two separate fiber bundles (Figure 1a). Each fiber traverses $K$ voxels, where $K$ is a function of fiber length. One of the two fibers traverses a localized area of white matter pathology, such as a circumscribed lesion. We assume that pathology is circumscribed to one, or a few, of the $K$ voxels traversed by the pathological fiber, resulting in a reduction in the FOD lobe integral from $v$ to $v_{f} \geq 0$ only in these voxels. This reduction, in addition to a possible rotation of the FOD orientation due to pathology, can partially or fully obstruct streamline propagation at the site of 
pathology, resulting in fewer streamlines traversing the full length of the pathological fiber, compared to the normal fiber. In our model, the probability of a streamline traversing the full length of the pathological fiber is denoted with $p, 0 \leq p \leq 1$, where $p=1$ indicates absence of a lesion, while $p=0$ indicates complete streamline obstruction, due to severe pathology, such as fiber transection. While we fixed $p$ at 0.5 and $K$ at 5 in our original investigation (1), here we analyze a range of $K$ and $p$ values to characterize the effect of SIFT on varying fiber lengths $(K)$ and pathology severity $(p)$. We denote the total number of streamlines traversing the normal fiber with $N$. The total number of streamlines traversing the full length of the pathological fiber is therefore $N p$. We assume that $p$ is chosen such that $N p$ is an integer.

Analysis: We can study the performance of the SIFT method on our phantom analytically. SIFT will eliminate $n_{1} \in[0,1, \ldots, N]$ and $n_{2} \in[0,1, \ldots, N p]$ streamlines from the normal and pathological fiber, respectively, where $\vec{n}=\left(n_{1}, n_{2}\right)$ is chosen to minimize the cost function,

$$
f(\vec{n})=\left(\left(N p-n_{2}\right) \mu-v_{f}\right)^{2}+(K-1)\left(\left(N p-n_{2}\right) \mu-v\right)^{2}+K\left(\left(N-n_{1}\right) \mu-v\right)^{2} .
$$

In the above cost function, the SIFT proportionality coefficient is denoted with $\mu$ and given by $\mu=\left(2 K v-v+v_{f}\right) /\left(K\left(N p+N-n_{1}-n_{2}\right)\right)$. In practice, the cost function is minimized approximately using gradient descent (2). Here, relaxing the integrality constraint on $\vec{n}$ enables exact minimization. Solving the pair of equations $\nabla f(\vec{n})=\overrightarrow{0}$ and showing that the Hessian matrix has a positive determinant yields,

$$
\left(n_{1}^{*}, n_{2}^{*}\right) \triangleq \operatorname{argmin} f(\vec{n})=\left(N-\frac{K N p v}{v_{f}-v+K v}, 0\right) .
$$

Given that $n_{1}^{*}$ is not necessarily an integer in the above equation, we apply the integer remapping,

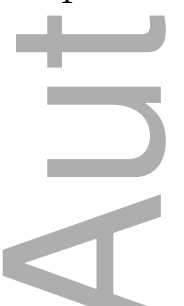

$$
n^{*} \leftarrow\left\{\begin{array}{l}
\left\lfloor n^{*}\right\rfloor, f\left(\left\lfloor n^{*}\right\rfloor\right)<f\left(\left\lceil n^{*}\right\rceil\right) \\
\left\lceil n^{*}\right\rceil,
\end{array}\right.
$$

We have used an exhaustive search to verify that this integer remapping of the optimal non-integer minimum (red and blue curves in Figure $2 \mathrm{c}$ ) yields an excellent approximation of the true integer minima (dashed black curves) of the cost function. 
Under SIFT with the modifications proposed by Smith and colleagues (3), the connectivity of the pathological and normal fiber is given by $N p \mu$ and $\left(N-n_{1}^{*}\right) \mu$, respectively. Following these authors, we compared the connectivity of each of the two fibers between a hypothetical patient $(p<1)$ and a hypothetical healthy control $\left(p=1, n_{1}^{*}=0, \mu=v / N\right)$ for the case of $N=40$ and various fiber lengths ( $K=5,10,40)$.

For the hypothetical patient's normal (blue) and pathological (red) fiber, we show: i) connectivity measured with the raw streamline count (Figure 2b) and after filtering with SIFT (Figure 2c); and, ii) percentage change in connectivity between the patient and control, defined as (control - patient) / control $\times 100 \%$. The latter measure is henceforth referred to as the percentage change and can be interpreted as a putative effect size for the patient-control difference.

Figure $2 \mathrm{~b}$ exemplifies several paradoxical behaviors of the SIFT method.

- First, the hypothetical patient's normal fiber shows paradoxical decreases in connectivity, which are often equal in percentage change to the pathological fiber. This is nonsensical and can result in wrongly concluding that pathology is present in a normal fiber. For example, for a fiber length of $80 \mathrm{~mm}$, connectivity in the normal fiber is equal to the connectivity in the pathological fiber for all levels of pathology severity (red and blue curves overlap).

- Second, the percentage change in connectivity is erratic and not monotonic in $N p$. This means that the percentage change can paradoxically increase with lessening severity of pathology.

- Third, the percentage change decreases with fiber length, suggesting a bias toward detection of pathology in short fibers.

- Finally, the percentage change is markedly lower after SIFT filtering compared to the raw streamline count. For example, despite a $100 \%$ reduction in the raw streamline count, the percentage change in connectivity under SIFT is less than $1.5 \%$ for the $80 \mathrm{~mm}$ fiber. This can obscure effect size estimates for between-group comparisons and enormously reduce statistical power to detect connectivity differences as a consequence.

These concerns are not idiosyncratic to our pathological fiber phantom. The paradoxical behaviors of SIFT are also evident for: i) a larger number of streamlines ( $N=1000$, Figure $2 \mathrm{~d}$ ); ii) pathological fibers in which the FOD integral is reduced in $20 \%$ of the FOD lobes; and, iii) a larger fiber phantom comprising 
four normal and four pathological fibers, with the value of $p$ differing between the pathological fibers to introduce heterogeneity in pathology severity.

Figure $2 \mathrm{e}$ shows that relaxing the integrality constraint on $n_{1}^{*}$ yields monotonicity of the percent change as a function of $\mathrm{Np}$. Alternative streamline post-processing methods based on continuous weighting schemes $(6,7,8)$ might therefore furnish connectivity measures that alleviate the first two paradoxical behaviors listed above. We limit this note to the SIFT method.

Interpretation: The premise of SIFT is that FOD integrals and streamline counts should match globally for all FOD lobes. We contend that this premise can breakdown in the presence of some pathologies. Transecting, crushing or intoxicating a fiber bundle at any circumscribed site along its trajectory will hamper neural signaling between the two regions interconnected by the fiber, thereby warranting a reduction in the fiber's connectivity strength, even if the resulting connectivity strength (i.e. streamline count) does not match the majority of FOD lobes traversed by the fiber. The adage $a$ chain is only as strong as its weakest link provides a useful analogy here. SIFT is inconsistent with this adage because it seeks to adjust the strength of the chain in such a way that chain strength and link strength are consistent for all links in the chain. However, measurement of interregional connectivity should remain faithful to the adage. In particular, neural signaling should be able to be exclusively dictated by the weakest link along a fiber bundle; namely, the lesion location. Just like breaking any single individual link in a chain breaks the chain's ability to transmit a force, transecting a fiber bundle at any point disrupts the fiber's capacity to transmit neural signals. This suggests that the connectivity strength of a severed fiber must be zero, or some minimum value, irrespective of whether the majority of FOD lobes comprising the fiber are not consistent with a streamline count of zero. Seeking to artificially impose consistency between FOD lobes and streamline counts can conceal the effect of pathology, just as it would conceal the weakest link in a chain.

While the pathology simulated here is generic in nature, to provide a concrete example, it could model Wallerian degeneration owing to a focal traumatic brain injury. Under Wallerian degeneration, the portion of the fiber stump that is distal to the injury undergoes granular disintegration of its axonal cytoskeleton, whereas the proximal portion remains intact and electrically excitable (7). The diffusion signal measured within the proximal portion of the fiber could thus differ markedly from the signal at 
the injury site as well as the signal within the distal fiber portion. Seeking to impose correspondence between the diffusion signals and the streamline count under these circumstances is therefore an illconceived objective in our opinion. Myelin abnormalities and other connectome pathologies (8) associated with psychiatric disorders such as schizophrenia can yield comparable circumstances in which pathology is constrained to a circumscribed fiber segment.

We recommend that if the SIFT method is used, it should be used cautiously when applied to pathological connectomes, irrespective of whether the modifications proposed by Smith and colleagues are adopted. We call to question the premise of seeking to impose global consistency between interregional streamline counts and local aspects of the diffusion signal in diseased connectomes. However, it is important to remember that the paradoxical behaviors exemplified here remain hypothetical and their potential impact on empirical connectomes requires validation. We contend that a promising avenue toward enhancing the histopathological utility and interpretability of connectomes is to parameterize and sample streamline trajectories in terms of specific markers of brain tissue microstructure, such as neurite density and orientation dispersion (9), myelin water fraction (10) and extracellular free water (11).

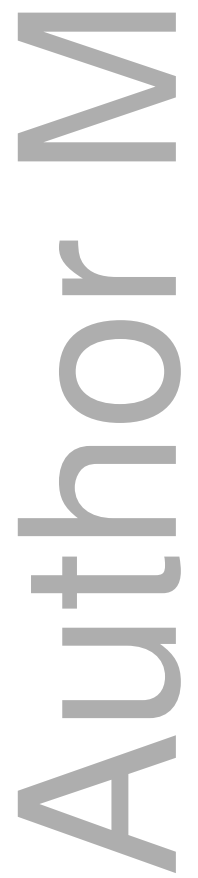


a

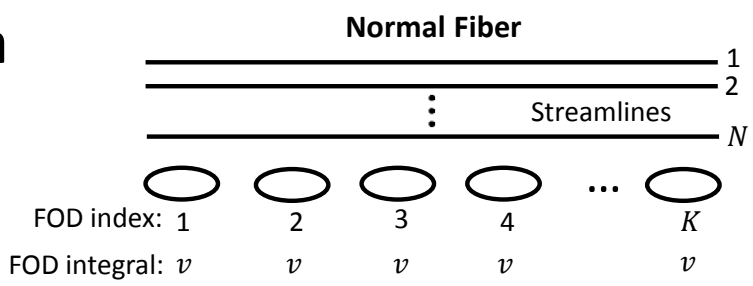

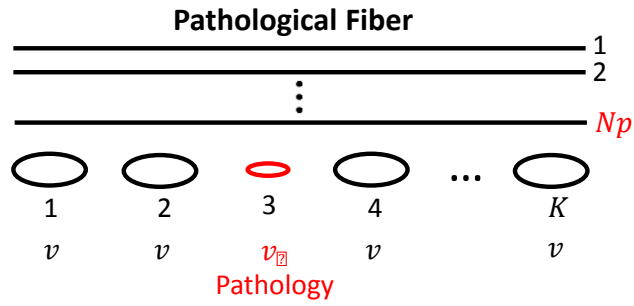
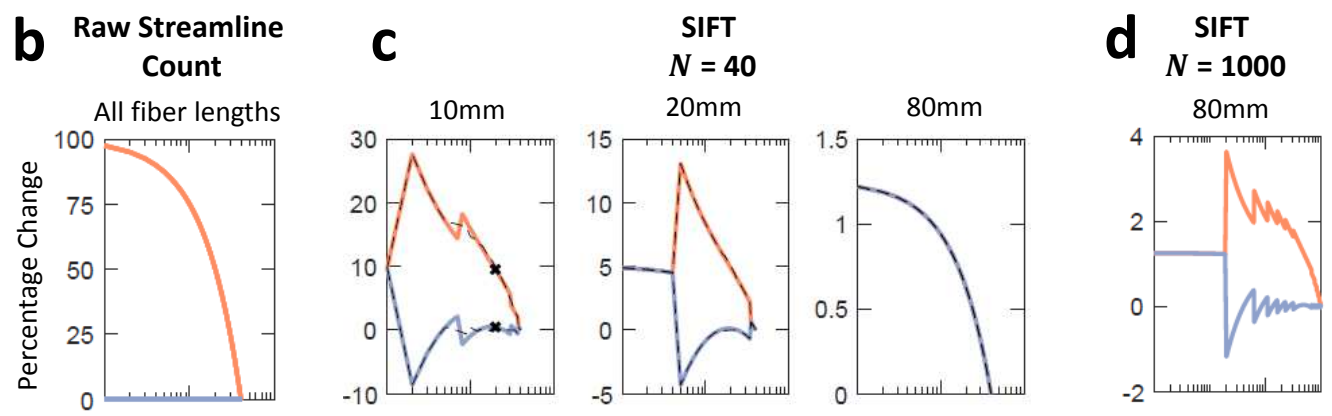

Non-Integer
Solution
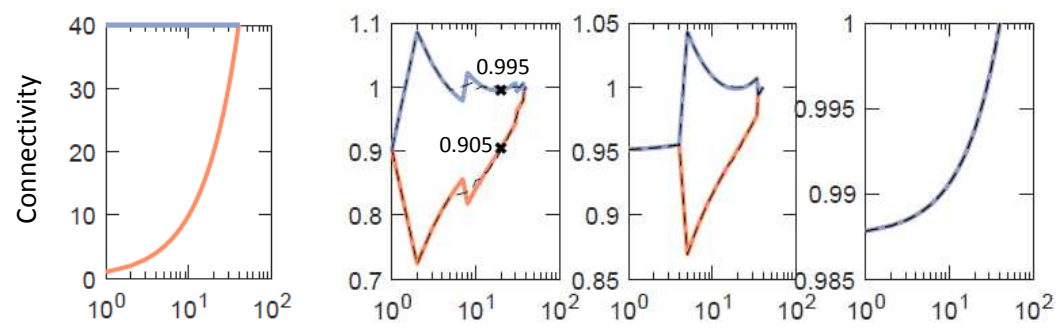
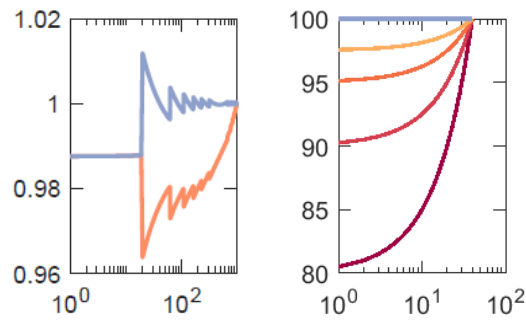

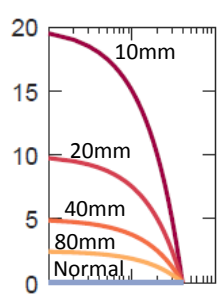

Number of Streamlines in Pathological Fiber $(N p)$

Pathological Fiber - Normal Fiber

- - - - - Integer Solution

Figure 1. Evaluation of the SIFT method in the presence of pathology. (a) Schematic of fiber bundle phantom. The phantom comprises two fibers, one of which traverses an area of localized white matter pathology. (b-e) Percentage change in connectivity between a hypothetical patient and a hypothetical control, defined as (control patient) / control $\times 100 \%$ (upper row) and connectivity as a function of the number of streamlines traversing the full length of the pathological fiber (lower row). Red: pathological fiber. Blue: normal fiber. Model parameters: $v=1, v_{f}=p, N p \in[1, \ldots, N]$ and $N=40$, unless indicated otherwise. Setting $v_{f}=p$ ensures that the pathological FOD integral is commensurate with the number of streamlines traversing the pathological fiber. Setting $v_{f}=0.5$ yielded comparable phenomena. (b) Connectivity measured based on the raw streamline count. (c) Same as (b), but connectivity is measured after SIFT filtering. Connectivity and percentage change become dependent on fiber length. Fiber length is indicated in millimeters $(\mathrm{mm})$ for a $2 \mathrm{~mm}$ voxel width $(K=5,10,40)$. Only blue curves are visible when blue and red curves overlap. Dashed black curves indicate an optimal integer solution determined with exhaustive search of the parameter space, whereas blue and red curves indicate integer remapping of the analytically derived non-integer optimal solution. Black crosses indicate the parameter combination considered in our original investigation ( $p=0.5, K=5$ ) and emphasized by Smith and colleagues. (d) Same as (b), but the 
number of streamlines traversing the normal fiber is increased to $N=1000$. (e) Relaxation of the integrality constraint on $n^{*}$.

\section{Abbreviations}

FOD: fiber orientation density

\section{References}

(1) Sarwar T, Kotagiri R, Zalesky A (2019) Mapping connectomes with diffusion MRI: Deterministic or probabilistic tractography? Magn Reson Med. 81(2):1368-1384

(2) Smith RE, Tournier J-D, Calamante F, Connelly A (2013) Spherical-deconvolution informed filtering of tractograms. Neurolmage. 67:298-312.

(3) Smith RE, Calamante F, Connelly A (2019) Mapping connectomes with diffusion MRI:

Deterministic or probabilistic tractography? Letter to the Editor. Magn Reson Med. 81(2):13681384

(4) Pestilli F, Yeatman JD, Rokem A, Kay KN, Wandell BA (2014) Evaluation and statistical inference for human connectomes. Nat Methods. 11(10):1058-1063.

(5) Daducci A, Dal Palu A, Lemkaddem A, Thiran J. COMMIT: Convex optimization modeling for micro-structure informed tractography. IEEE Tran Med Imaging. 2014;34(1):246-257.

(6) Smith RE, Tournier JD, Calamante F, Connelly A (2015) SIFT2: Enabling dense quantitative assessment of brain white matter connectivity using streamlines tractography. Neurolmage. 119:338-351.

(7) Vargas ME, Barres BA (2007) Why is Wallerian degeneration in the CNS so slow? Annual Review of Neuroscience. 30:153-79.

(8) Fornito A, Zalesky A, Breakspear M (2015) The connectomics of brain disorders. Nat Rev Neurosci. 16(3):159-72.

(9) Zhang H, Schneider T, Wheeler-Kingshott CA, Alexander DC (2012) NODDI: practical in vivo neurite orientation dispersion and density imaging of the human brain. Neurolmage. 61(4):1000-1016.

(10)Alonso-Ortiz E, Levesque IR, Pike GB (2014) MRI-based myelin water imaging: A technical review. Magn Reson Med. 73(1):70-81.

(11) Pasternak O, Sochen N, Gur Y, Intrator N, Assaf Y (2009) Free water elimination and mapping from diffusion MRI. Magn Reson Med. 62(3):717-730. 
a



Pathological Fitor
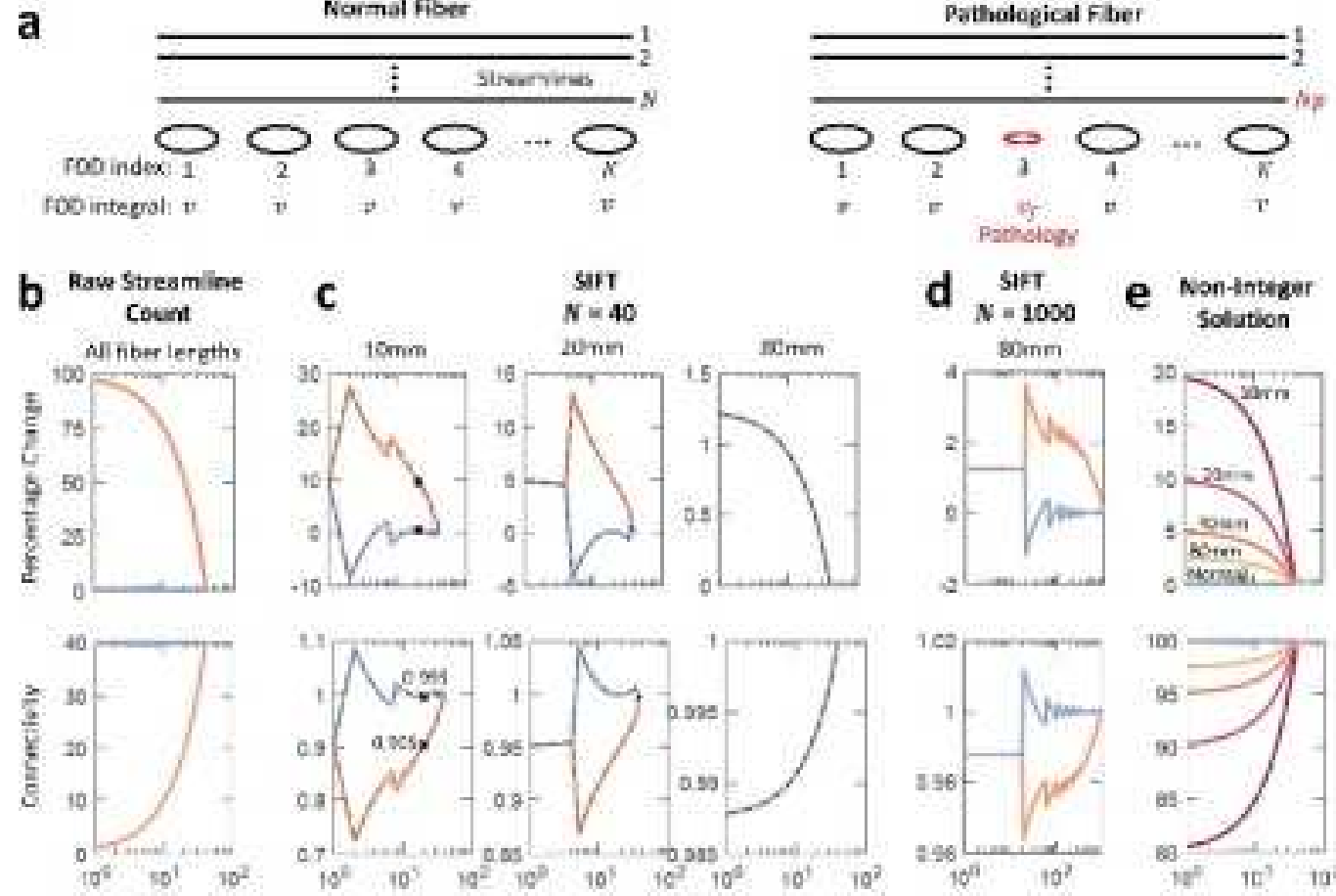
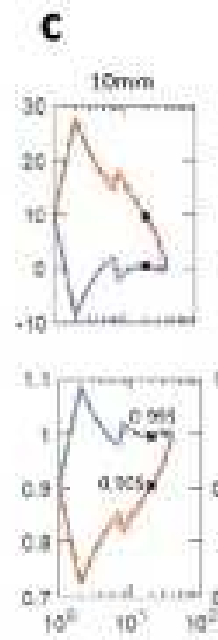

SIF $N=40$ $20 \mathrm{men}$
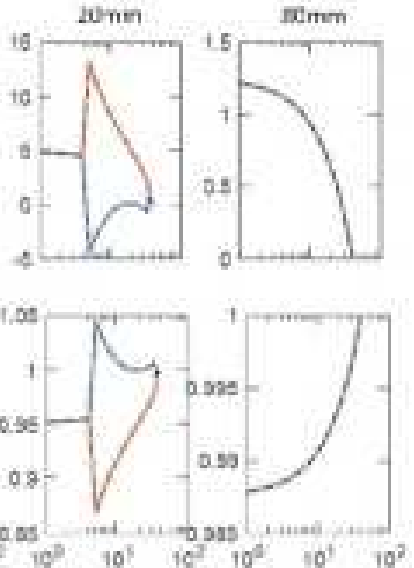

Number of Strmanlines in Pathological Aber iNo

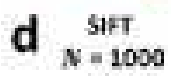

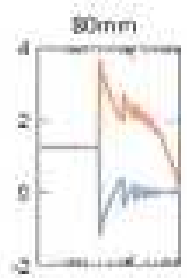

e Non-Integer
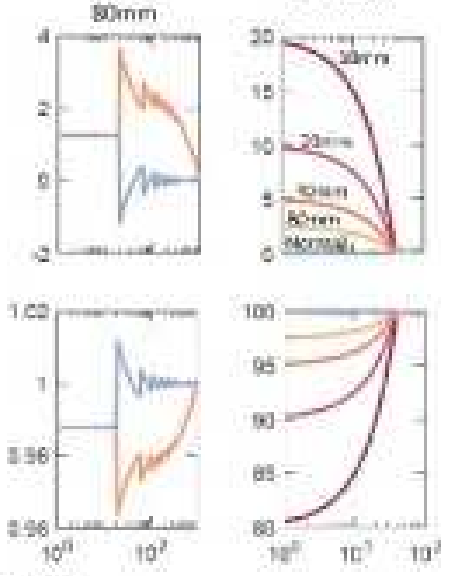

- easiclopicat Fiber Normal Fiber

-.......- hacger Soution

mrm_28037_f1.tif 


\section{University Library}

\section{- M M I N E R VA A gateway to Melbourne's research publications}

Minerva Access is the Institutional Repository of The University of Melbourne

Author/s:

Zalesky, A;Sarwar, T;Ramamohanarao, K

Title:

A cautionary note on the use of SIFT in pathological connectomes

Date:

2019-10-21

Citation:

Zalesky, A., Sarwar, T. \& Ramamohanarao, K. (2019). A cautionary note on the use of SIFT in pathological connectomes. MAGNETIC RESONANCE IN MEDICINE, 83 (3), pp.791-794. https://doi.org/10.1002/mrm.28037.

Persistent Link:

http://hdl.handle.net/11343/286523 